DISCLAIMER

This document was prepared as an account of work sponsored by an agency of the United States Government. Neither the United States Government nor the University of California nor any of their employees, makes any warranty, express or implied, or assumes any legal liability or responsibility for the accuracy, completeness, or usefulness of any information, apparatus, product, or process disclosed, or represents that its use would not infringe privately owned rights. Reference herein to any specific commercial product, process, or service by trade name, trademark, manufacturer, or otherwise, does not necessarily constitute or imply its endorsement, recommendation, or favoring by the United States Government or the University of California. The views and opinions of authors expressed herein do not necessarily state or reflect those of the United States Government or the University of California, and shall not be used for advertising or product endorsement purposes. 


\section{ATOMIC SCALE SIMULATIONS OF ARSENIC ION IMPLANTATION AND ANNEALING IN SILICON}

M.-J. Caturla ${ }^{2}$, T. Díaz de la Rubia ${ }^{1}$, M. Jaraiz ${ }^{2}$ and G.H. Gilmer ${ }^{3}$

${ }^{1}$ Lawrence Livermore National Laboratory, L-268, Livermore, CA-94550

${ }^{2}$ Dept. E. y Electrónica, Universidad de Valladolid, Valladolid, Spain

${ }^{3}$ AT\&T Bell Laboratories, Rm. 1E332, 600 Mountain Ave., Murray Hill, NJ-07974

\section{ABSTRACT}

We present results of multiple-time-scale simulations of 5, 10 and $15 \mathrm{keV}$ low temperature ion implantation of arsenic on silicon (100), followed by high temperature anneals. The simulations start with a molecular dynamics (MD) calculation of the primary state of damage after 10ps. The results are then coupled to a kinetic Monte Carlo (MC) simulation of bulk defect diffusion and clustering. Dose accumulation is achieved considering that at low temperatures the damage produced in the lattice is stable. After the desired dose is accumulated, the system is annealed at $800^{\circ} \mathrm{C}$ for several seconds. The results provide information on the evolution for the damage microstructure over macroscopic length and time scales and affords direct comparison to experimental results. We discuss the database of inputs to the MC model and how it affects the diffusion process.

\section{INTRODUCTION}

The manufacturing of VLSI devices is based on the implantation of dopants and recovery of the lattice structure by annealing at high temperature [1]. During annealing the defects produced by the implantation interact with the dopants. This process results in transient enhanced dopant diffusion (TED) $[2,3,4]$. It has been observed that at high dose and energy extended defects are produced that, as they evaporate induce transient enhanced diffusion [5]. The critical number of displaced atoms necessary to produce these extended defects is reached at different doses for different ions masses and energies [6]. The minimum dose for $\{311\}$ defect formation with $40 \mathrm{keV} \mathrm{Si}$ implantation in $\mathrm{Si}$ is approximately $5 \times 10^{12} \mathrm{~cm}^{-2}$ [4]. This minimum dose has been observed to increase as the energy of the ion decreases. Therefore, at very low energies and dose $\{311\}$ defects are not present and still transient enhanced diffusion occurs [7]. Doses as low as $10^{12} \mathrm{~cm}^{-2}$ for $180 \mathrm{keV} \mathrm{Si}$ implant induce transient $B$ diffusion. However, for doses lower than $10^{13} \mathrm{~cm}^{-2}$ the enhancement in the diffusivity is almost constant [8].

Modeling of these.phenomena requires methods that allow for long time scale calculations as well as large sizes. Recently Jaraiz et al. have shown that a hybrid between binary collision approximation models and Monte Carlo simulations gives results in very good agreement with experiments for $40 \mathrm{keV} \mathrm{Si}$ implantation in (001) $\mathrm{Si}$ and posterior annealing at high temperatures [9]. In this paper we describe a coupling between molecular dynamics simulations and Monte Carlo models in order to give a better insight to the mechanisms involved in the production and diffusion of defects in Silicon. We will present first the results obtained for single As cascade events on Silicon at different energies. We explain how the connection with Monte Carlo simulations is made and the parameters 
considered in this simulation. The last part of this communication will be a study of the influence of the ion dose in the recombination factor in the bulk at high temperatures.

\section{INITIAL COLLISION STAGE: MOLECULAR DYNAMICS SIMULATION}

In the MD simulations described, we employ the Stillinger-Weber (SW) potential for Silicon [10]. In agreement with ab-initio simulations [11], this potential predicts the $<110>$ split dumbbell configuration as the lowest energy structure for neutral self interstitial [12], although the exact details of the configuration of the dumbbell are slightly different in these two models. In addition, to describe short range interactions we have connected the Stillinger-Weber potential to the Universal potential of Biersack, Littmark and Ziegler [13], following the approach by Gärtner et al. [14]. The energy losses due to inelastic collisions with the electrons in the system are simulated through the Lindhard model [15]. The boundary layers of the simulation box are coupled to a thermal bath to avoid re-entering of the energy in the system. The simulations were performed in a CRAY T3D MPP parallel computer at LLNL. The code employs PVM message passing routines [16] for communication between processors. The CPU time with 128 processor elements is of $2 \mathrm{~s}$ per time step for a million atom system.

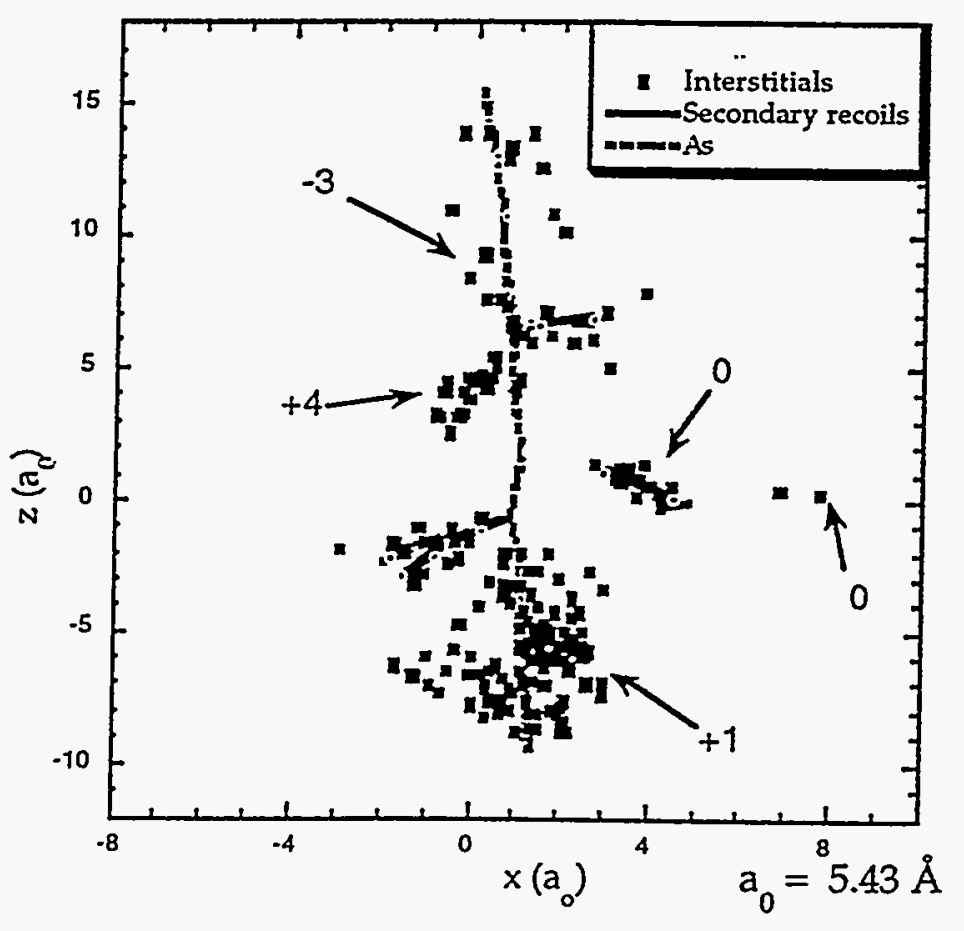

Figure 1. As $5 \mathrm{keV}$ on $\mathrm{Si}$ at $300 \mathrm{~K}$ after $10 \mathrm{ps}$.

We have studied the interaction of As ions in Silicon at room temperature and different energies of the projectile. In Fig.1 we present the damage produced in the silicon target 10ps after the impact of a $5 \mathrm{keV}$ As ion. The dashed line represents the trajectory of the ion. The damage is described in terms of vacancies and interstitials. An atom that moves more than the first nearest neighbors distance $(2.35 \AA$ in silicon) is considered an interstitial and the initial position of this atom is a vacancy, unless the atom is in half a nearest neighbor distance of a vacancy, in which case we consider it as a replacement. In Fig.1 the open circles represent the vacancies and the squares are the interstitials. We can observe in this figure that unlike in the case of a metal [17], in semiconductors there are almost no replacement collision sequences and the group of defects produced far from the ion trajectory are due to channeling of secondary recoils, (represented by solid lines in Fig.1). The vacancies and interstitials can be clustered into groups of defects. We 
will consider that two defects, vacancy or interstitial, belong to the same cluster if the distance between them is less than the cut off for the Stillinger-Weber potential, $3.77 \AA$. Each cluster will contains a certain number of vacancies and interstitials so the deficit or excess of atoms in a cluster can be obtained. In Fig. I we present some of the excess $(+)$ or deficit $(-)$ of atoms for the different defect clusters. These numbers are always very small $(<5)$ for all the cases studied. The maximum cluster size increases with the energy of the ion, going from $\sim 60$ atoms for a $2 \mathrm{keV}$ As impact to $\sim 500$ for $15 \mathrm{keV}$. In all cases the fraction of defects in clusters higher that 10 is $\sim 70 \%$. Only $\sim 10 \%$ of the total number of defects in the lattice are isolated vacancies or isolated interstitials, however the total number increases with energy. These numbers have been obtained from an average of at least three independent events at each energy. The number of defects produced at 10ps is approximately twice the value predicted by binary collision models [18]. The slope obtained from plotting the number of interstitials as a function of damage energy is: $=55 \mathrm{keV}^{-1}$ $2 \times 0.8 / 2 \mathrm{E}_{\mathrm{d}}$, where $\mathrm{E}_{\mathrm{d}}=15 \mathrm{eV}$ is the threshold displacement energy for defect production in $\mathrm{Si}$.

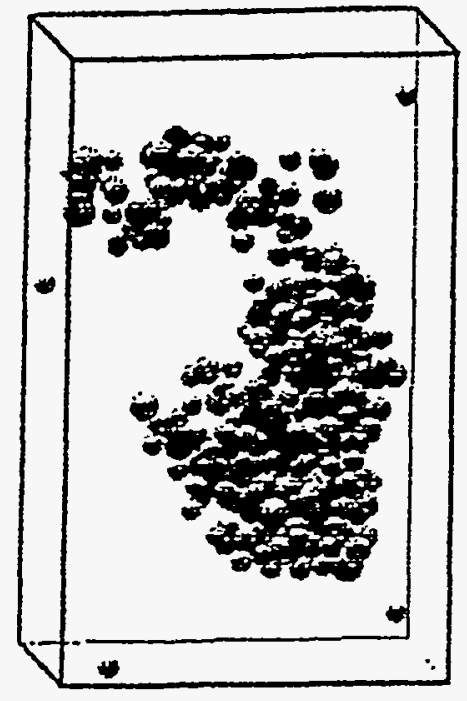

(a)

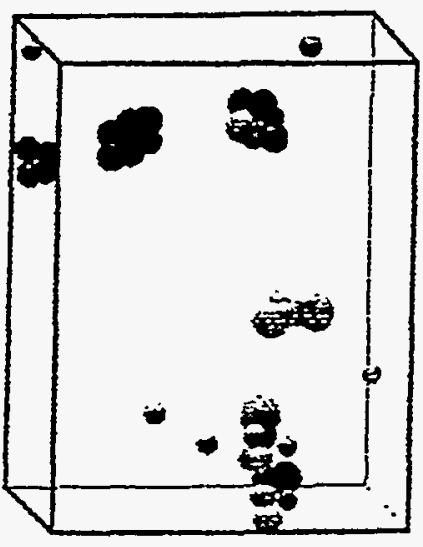

(b)

Figure 2. Atoms with $\mathrm{Ep}>0.2 \mathrm{eV}$ (a) 'amorphous' pocket from a $5 \mathrm{keV}$ As in Si at RT, (b) after annealing during $0.5 \mathrm{~ns}$ at $800^{\circ} \mathrm{C}$

We can also look at the damage in terms of those atoms that have a potential energy $0.2 \mathrm{eV}$ above the ground state. This criteria is not so strict as the one described above, and we can observe here all the distorted atoms due to the ion impact. As we discussed in previous papers [19], as a result of the high energy cascades, the damage produced is amorphous like, at least for high mass ions. This phenomena explains the discrepancy between the number of displaced atoms obtained in this work and those predicted by BCA models. The ion impact induces a localized high energy region, that results in the production of amorphous pockets, containing more displaced atoms that just those predicted by simple binary collisions. In order to study the behavior of these disordered regions under high temperatures, we extract from the $5 \mathrm{keV}$ As cascade an amorphous pocket, like the one we present in figure 2(a), and we anneal it at high temperature, $800^{\circ} \mathrm{C}$. After only $200 \mathrm{ps}$ the pocket has been recrystalized and only a few number of point defects and clusters of defects are left. In this plot the dark greys represent those atoms under tensile stress, therefore, correspond to those atoms surrounding a vacancy, while the light greys represent the atoms under compressive stress related to interstitials. Only $\sim 1 \%$ of the initial number of atoms in the amorphous pocket are left in the crystal after recrystallization at high 
temperature. Therefore, it seems reasonable to assume in the Monte Carlo model simple recombination of the $\mathrm{V}$ and I present in the amorphous pockets at high temperature. Only the excess or deficit of atoms in the clusters will remain. For the simulation of damage accumulation of cascades it is necessary to study the stability of these amorphous zones at low temperatures. We have observed that for RT or lower the amorphous pockets seem to reach stability after only a few picoseconds, and remain stable, at least for the $0.5 \mathrm{~ns}$ simulated [20].

Although the criteria described above for vacancies and interstitials is a simple way of representing the damage, it provides a useful bridge between the molecular dynamics results and the simulation of diffusion of defects by Monte Carlo.

\section{DAMAGE ANNEALING: MONTE CARLO SIMULATIONS}

We use Monte Carlo simulations to study the long-time evolution of the As-induced damage. The parameters used for this calculation are all extracted from molecular dynamics simulations with the Stillinger Weber potential. The values used for the diffusivity, migration energies and binding energies for clusters of vacancies and interstitials of different sizes are described in Table I (see [12] for more details).

Table I. Parameters for MC simulation. Obtained from MD calculations [12]

\begin{tabular}{|l|c|c|c|c|c|}
\cline { 2 - 6 } \multicolumn{1}{c|}{} & $\begin{array}{c}\text { Diffusivity } \\
\left(\mathrm{cm}^{2} / \mathrm{s}\right)\end{array}$ & $\begin{array}{c}\text { Binding } \\
\text { Energy } \\
\text { Size 2 }\end{array}$ & $\begin{array}{c}\text { Binding } \\
\text { Energy } \\
\text { Size 3 }\end{array}$ & $\begin{array}{c}\text { Binding } \\
\text { Energy } \\
\text { Size 4 }\end{array}$ & $\begin{array}{c}\text { Binding } \\
\text { Energy } \\
\text { Size 5 }\end{array}$ \\
\hline Vacancy & $0.001 \exp (-0.43 / \mathrm{KT})$ & 0.62 & 0.78 & 1.2 & 1.82 \\
\hline Interstitial & $0.01 \exp (-0.9 / \mathrm{KT})$ & 1.6 & 2.25 & 1.29 & 2.29 \\
\hline
\end{tabular}

We observe that for small sizes, the binding energies of interstitial clusters do not increase or decrease monotonicaly, but rather fluctuate. For clusters of size $n>5$ we consider binding energies of $1.5 \mathrm{eV}$ for $\mathrm{V}$ and $2.1-1.2(\sqrt{n}-\sqrt{n-1}) \mathrm{eV}$ for $\mathrm{I}$ clusters.

The initial distributions of vacancies and interstitials are obtained from the MD simulations described above. To simulate dose accumulation, we assume no intercascade recombination between vacancies and interstitials, an approach supported by the studies explained above about the stability of amorphous pockets at low temperatures.

We simulate the diffusion of defects during annealing at $800{ }^{\circ} \mathrm{C}$ and different doses, for $5 \mathrm{keV}$ As implantation in Silicon. In Fig.3 we present the average size of the $\mathrm{V}$ and I clusters as a function of time, for a dose of $5 \times 10^{12} \mathrm{~cm}^{-2}$. Observe that the average size is always very small. Due to the low doses and energies, and the fluctuations in the binding energies for I, no extended defects are observed in this case, in agreement with the experiments [7]. Note that the maximum I cluster sizes are reached at about 0.1 secs, and contain an average of only 4 interstitials, i.e., the average between 3 and 5 I clusters, which are those with the largest binding energy. 
In Fig.4 we show the

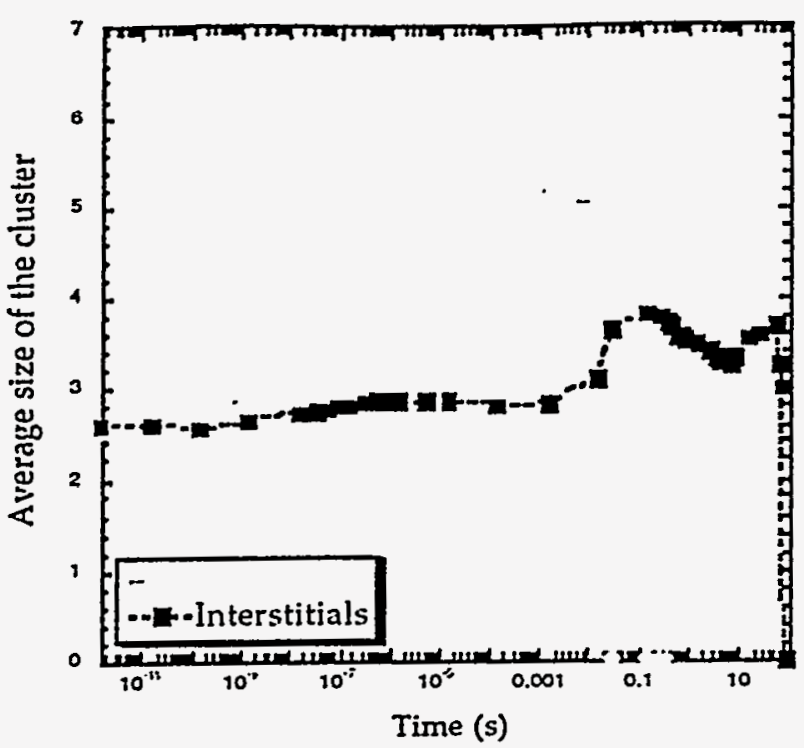

Figure 3. Average size of $\mathrm{V}$ and I clusters as a function of annealing time, for $5 \times 10^{12} \mathrm{~cm}-2$ and $800^{\circ} \mathrm{C}$. As $5 \mathrm{keV}$ in Si.

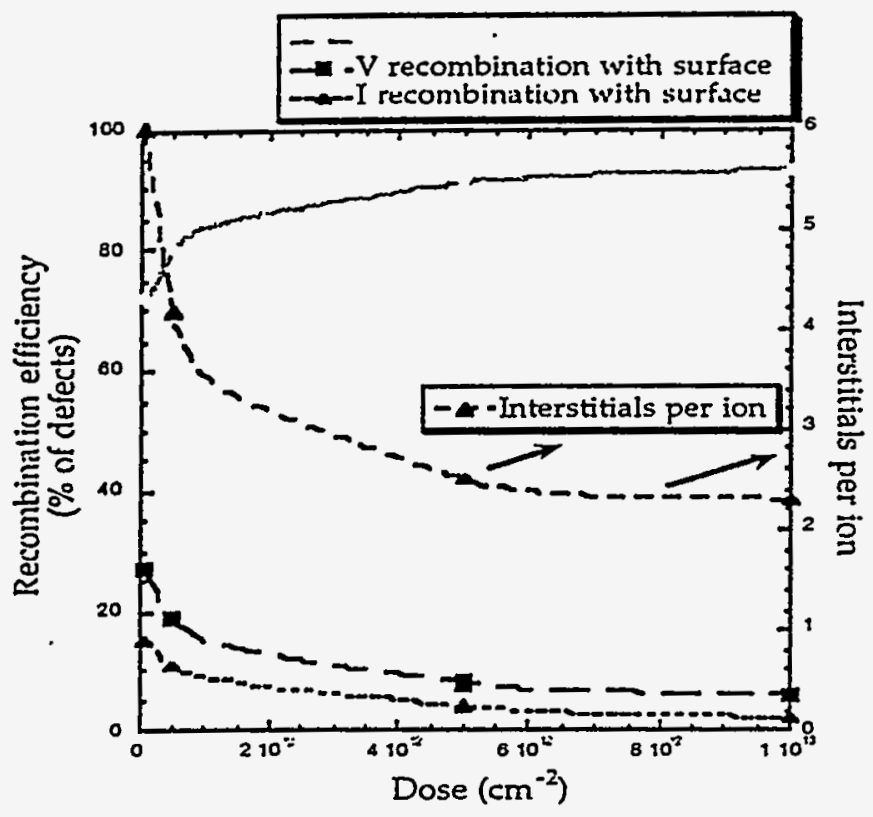

Figure 4. Recombination efficiency as a function of dose in the bulk and in the surface for $\mathrm{V}$ and $\mathrm{I}$

for annealing of damage resulting from As implantation and not $B$, provide some initial, indirect validation of such a model.

\section{SUMMARY}

We have used MD simulations coupled to a simple kinetic MC diffusion model to study implantation and annealing of $\mathrm{As}$ into $\mathrm{Si}$ at low energy and low dose. We observe from the MD results that the number of isolated defects produced is only a

recombination efficiency of defects in the bulk and in the surface as a function of dose. For low doses more $\mathrm{V}$ and $\mathrm{I}$ arrive to the surface before recombining in the bulk. Due to the different migration energies of $\mathrm{V}$ and $I$ and low energies of implantation considered in this study, the fraction of $V$ recombining with the surface is always bigger than the I fraction. Also shown in this figure is the number of I left in the system after all vacancies have either recombined in the bulk or reached the surface. This number decreases from a value of +6 at low dose $\left(10^{10}\right.$ $\mathrm{cm}^{-2}$ ) to a constant value of $\approx+2$ at doses larger than about $6 \times 10^{12} \mathrm{~cm}^{-2}$. This number, also known as the " + " number is then responsible for transient dopant diffusion. The observed decrease in the + number with dose and its approach to a constant at high dose is interesting. As we noted above, the enhancement in the diffusivity for $B$ transient diffusion experiments is constant at low doses and increases almost linearly above $10^{13} \mathrm{~cm}^{-2}$ [8]. This result may be consistently explained by taking into account the fact that although the total number of defects produced increases with dose, the actual number of defects available for diffusion of the dopant per ion (i.e., the + number) decreases with increases dose up to $\approx 10^{13} \mathrm{~cm}^{-2}$. The simulation results presented here, although obtained 
$10 \%$ of the total number of defects. Most of the damage is concentrated into amorphous-like pockets. This pockets anneal at $800^{\circ} \mathrm{C}$ producing point defects and small clusters. We use the $M D$ results as input for $M C$ annealing of the damage. Simulations at different doses show a decrease in the bulk recombination efficiency relative to surface recombination as the dose decreases. In addition, we observe that the number of self interstitials left in the lattice after all the vacancies disappear decreases from a value of +6 at low doses to a constant value of $\approx+2$ at doses larger that $\approx 6 \times 10^{12} \mathrm{~cm}^{-2}$.

\section{ACKNOWLEDGMENTS}

This work was performed under the auspices of the U.S. Department of Energy by Lawrence Livermore National Laboratory under Contract No. W-7405-Eng-48 and by AT\&T Bell Laboratories. One of us (M.J.C.) thanks the Juan Gil Albert Association of Alacant for partial support.

* Permanent address: Dept. de Física Aplicada, Universtitat d'Alacant, Spain

\section{REFERENCES}

[1] Handbook of Ion Implantation, Ed. by J.F. Ziegler (North Holland, Amsterdam, 1992)

[2] T. Motooka and O.W. Holland, Apply. Phys. Lett. 61 (1992) 3005

[3] K.S. Jones, H.G. Robinson, J. Listebarger, J. Chen, J. Liu, B. Herner, H. Park, M.E. Law, D. Sieloff, J. A. Slinkman, Nucl. Instrum. and Methods, B96 (1995) 196

[4] D.J. Eaglesham, P. A. Stolk, H.-J. Gossman, and J.M. Poate, Apply. Phys. Lett. 65 (1994) 2305

[5] P. A. Stolk, H.J. Gossmann, D.J. Eaglesham, J.M.Poate, Nud. Instrum. and Methods, B96 (1995) 187

[6] R. J. Schreutelkamp, J.S. Custer, J.R. Liefting, W.X. Lu and F.W. Saris, Materials Science Reports, 6 (1991)

[7] L.H. Zhang, K.S. Jones, P.H. Chi, D.S. Simons, Apply. Phys. Lett 67 (1195) 2025

[8] P.A. Packan and J.D. Plummer, Apply. Phys. Lett 56 (1990) 1787

[9] M. Jaraiz, G.H. Gilmer, J. M. Poate and T. Diaz de la Rubia, unpublished.

[10] F.H. Stillinger and T.A. Weber, Phys, Rev. B31 (1985) 5262

[11] J. Zhu, L. Yang, C. Mailhiot, T. Diaz de la Rubia, G.H. Gilmer, Nucl. Instrum. and Methods $B$, in press

[12] G.H. Gilmer, T. Diaz de la Rubia, D. Stock and M. Jaraiz, Nucl. Instrum. and Methods B102 (1995) 247

[13] J.P. Biersack and J.F. Ziegler, Nucl. Instrum. and Methods. 194 (1982) 93

[14] K. Gärtner, Nucl. Instrum. and Methods B102 (1995) 183

[15] J. Lindhard and M. Sharff, Phys. Rev. 124, 128 (1961)

[16] PVM3 Users Guide and Reference Manual. ORNL/TM-12187, May 1993

[17]T. Diaz de la Rubia, R.S. Averback, R. Benedek and W.E. King, Phys. Rev. Lett. 59 (1987) 1930

[18] P. Sigmund, Apply. Phys. Lett., 14 (1969) 114

[19] M.-J. Caturla, T. Diaz de la Rubia, L.A. Marques, G.H. Gilmer, unpublished.

[20] T. Diaz de la Rubia and G.H. Gilmer, Phys. Rev. Lett. 74 (1995) 2507 